\title{
He poroporoaki ki te rangatira nā tana irāmutu
}

\section{He kupu whakataki:}

I te 29 o ngā rā o Whiringa ā-nuku, 2010, i mate mai tētehi o ngā tino kaumātua nō te kāinga nei, nō Te Tahaaroa. Ko te iwi ko Waikato, ko te hapū ko Ngāti Mahuta (ki te tai hauāuru). Ko tōna marae ko Te Kōraha, ā, ko Wharetoroa Robert (Bob) Kerr tōna ingoa. He pou whakakikiwā, he teo herenga waka, he rākau tau matua nō roto tonu mai i te rohe o Tainui. I taetae atu te iti, te rahi, me kī rā, te hārakerake ki te tuku mihi, tuku poroporoaki me te whakatakoto kōrero ki te marae ātea mō te rangatira nei i te wā i takahia ai e ia te mata o te whenua.

I tae atu te taniwhā hikuroa o te motu nei, arā, ko te Kīngi Māori, ko Kīngi Tūheitia, nāna anō i whakakanohi mai ai ngā Kīngi kua riro atu ki te pō, ki a Kīngi Pōtatau, Kīngi Tāwhiao, Kīngi Mahuta, Kīngi Te Rata, Kīngi Korokī tae atu rā ki te Kuīni Māori, ki tōna whaea ki a Te Âtairangikaahu. Kauanuanu ana nā te mea he tuatahi tēnei ki te marae o Te Koraha, arā, te taenga mai o te Kīngi Māori a Kīngi Tūheitia. Nō reira, nō te hapū nei nō Ngāti Mahuta ki te hauāuru te maringanui i te mea e whakatinana ana ia i te whakatauki $\overline{1}$, he kōtuku rerenga tahi.

Whai mai i te Kīngi ko ngā rangatira nō Waikato ki tua whenua, nō Tauranga ki tua moana, nō Mataatua nō te whārua o Ruātoki, nō Ngātokimatawhaorua nō Te Rarawa me Ngāti Kahu, anā, nō ngā mātā waka anō hoki. Kākāriki pai ana te papa nekehanga ki ngā hoa ō mua i mau i a rātou ngō rātou nā tae, arā, ngā hoa kaitākaro rīki nō te Karapu Rīki o Taniwharau me ngētehi nō te Tīma Rīki Māori. Haruru pai ana 
te marae i ngā tini haka a ngā rōpu hoe waka, arā, ngā kaihoe waka ama o te motu, ngā reo rōreka o ngā tamariki nō te Wharekura o Ngā Taiatea, ngā kupu waiwaiā i whakatakotohia ki ngā rekereke o te koroua e ngā momo tāngata nō ngā whare wānanga, nō ngā wānanga, nō ngā kuratini, nō ngā kura, nō ngā hau e whā, nō ngā moutere o Te Moana Nui a Kiwa, nō Tonga, nō Whìti, tae atu rā ki ngā matamata huanga nō ngā moutere o Hawai'i.

Ko te kaituhi nei te kaikōrero tuatahi i te taha o te tangata whenua, nā te kaituhi te huarahi i para. Nei rā te reo wāwāhi, ngā kupu whakamihi me ngā kōrero hono i whārikihia ki te marea, ki mua tonu $\mathrm{i}$ te iwi $\mathrm{i}$ tae atu ki te tangihanga $\mathrm{o}$ Wharetoroa Robert (Bob) Kerr, ki runga i te marae o Te Kōraha.

Moe mai e Koro, moe mai rā.

\section{Te Whaikōrero:}

E Io o te rangi

Ka tō, ka tō

Ka tōia ki uta, ka tōia ki tai

Ka tau, ka tau

Ka tau ki runga, ka tau ki raro

He huatakitanga ka puea ake te pūrangiaho

Tēnei te waere

Tēnei te tārake

I te hāwatawata,

I te whakameme

Toroihi te mahukitanga

Uapare ana te manawapou

Manawapou roto

Manawapou waho

Ko te mauri ka tau

Ko te wairua ka tau

Ko te rongomaiwhiti ka tau

Taumārō ana 
Nau mai rā kia ahu atu ai ki ngā keokeonga o Awhero

Huke ana

Oke ana

Tuturu whakamaua kia tina, tina!

Haumi è

Hui ē

Tāiki è!

Tìhei mauri ora!

Kāore hoki te pō, kāore hoki te ao. Kimihia, rangahaua, kei whea rā koutou, ka ngaro nei e? Nō reira e te ika takoto a Tiki, takoto, takoto, takoto mai rā. Takoto mai ki tō waka, te waka i whakaritea mō koutou te tini, te mano kua riwha atu ki te pō uriuri, ki te pō tangotango, ki te pō i oti atu.

E koro e! Kua werohia tō kiri e te toki o aituā, tē taea te kaupare atu.

E koro e! Kua takahia e koe te ara o Hine-tūākirikiri, tē taea te waihape mai.

E koro e! Kua tae ngō turi ki ngō taringa, tē taea ngō wae te whātorotoro atu.

E koro e! Maranga mai, takatū ake, whakaoko mai!

Maranga mai ki te whakatau i tō Kīngi kua rarau mai nei, kua whakakatea mai nei $\mathrm{i}$ te nuku o te whenua, ki te whakaongaonga mai nei $i$ te rētōtanga me te rangawhāwhātanga o ngā taura here e rangitāmiro ana i a tātou i tēnei wā.

E koro, kua tae mai te Kīngi i runga anō i tō reo karanga e karapinepine mai ana $i$ te iwi nei, e whakarauika mai ana $i$ ngā mātā waka o te motu kia poroporoakitia, kia mihia, kia arohaina, kia whakaarohia koe. Nō reira, maranga mai!

Maranga mai e koro ki te whakatau i te Kīngi Māori e whakakanohi mai ana i ngōna tūpuna, arā, ngā Kīngi kua riro 
i te pō tae atu rā ki tōna whaea ki a Te Ātairangikaahu, arā, ki tō Kuīni Māori. E pāoro tonu ana tō reo waiwaiā i ngā pātūwatawata o Tūrongo, i te mahau o Mahinārangi, i te whakaruruhau o Kimikimi, i te papa marae hoki o Tūrangawaewae. Kua tāngia tonutia ki te whatumanawa ngāu kupu kōrero mō ngā Poukai. He pou nui whakaharahara hei tauwhiro i te pani, i te rawakore, i te pouaru. Nō reira nāu tō Ariki i tiaki, i poipoi, ā, i maimoā hoki.

Kua tae, kua tae, kua tae mai rā.

Ahakoa ka pēwhea te kupu kōrero, tōna pāpaku, tōna hōhonu, tōna whārahi, tōna whāiti, e kore koe e hoki mai, anā, kua huri tuarā mai koe. E ai ki te kōrero, he pae maunga tū te ao, tū te pō, engari kē ia he pae tangata ka ngaro, ka ngaro, ka ngaro.

Nō reira, haere ki Whakatere, haere ki Whakangaro, haere ki Whakaoti atu.

Ko rātou ki a rātou ngā akaaka o te rangi. Ko ngā akaaka o te whenua ki a tātou, e ora nei, e mana nei, e whai ana i ngā tapuwae o rātou mā, tēnā koutou, tēnā koutou, tēnā koutou katoa.

Tau ana i te tihi o Ōrangiwhao

Titiro whakararo ko Te Ohaaki runga Koraha

Hoki whakamuri ki ngā tai papaki o Honipaka

Tāpapa atu ki te pātaka ngaru o Paparoa

Whakarongo kau ana ki te tangi urumoana o te one

Whātoro atu ki te ruku hīnaki

Ki te roto o Tahaaroa rewa mai ki te Tahaaroa runga Āruka

Pikihia a Mangatangi te tihi okiokinga

Tare whakararo atu ki Muriwhenua

Ki ngā kōiwi mātua tūpuna

Ka tangi tonu te haruru moana

Huri tai āwhio ki te rohe o te Tahaaroa

He tangi nō mai rā anō 
He tangi nō ngā mātua tūpuna

Whakawhiti atu ki Te Ahurei ki te waka o Tainui

Ki Auau-ki-te-rangi, runga Maketu

Auē huri tai āwhio

Ko Ngāti Mahuta e!

Me wehi ki te Atua, whakahōnoretia tō tātou Kīngi Māori e whakamahana nei i te ahurewa rongomaiwhiti o ngōna mātua, o ngōna tūpuna, ki tōna hoa a Te Atawhai, ngā rāua tamariki, ngā rāua mokopuna, tae atu hoki rā ki te kāhui ariki whānui tonu. Kia tau iho ngā tauwhirotanga a te wāhi ngaro ki runga i a rātou katoa. Rire, rire, hau, paimarire.

Kei te whakaaro tonu ki a rātou mā kua hinga mai nei, kua hinga mai nā, kātahi anō a Te Auē ka ngaro atu i te tirohanga kanohi, kei te takoto, kua tīraha mai ki Tokikapu hei hoa haere mō te koroua nei, ko ngā parekawakawa kua pīkaungia mai e koutou, kia tangihia, kia mihia, kia poroporoakitia e tātou. Hei āwhea tēnei mea te rangikanetanga e mutu, kia māuru ake ai te aroha? Ka rere te hūpē me te roimata, ka kapo noa i tō hau e pā mai nei ki a au.

Āpiti hono, tātai hono, rātou te hunga wairua, te hunga mate ki a rātou, waiho mai ko tātou ngā kōkīkī, ngā poro tāwhaowhao kua rutua ki rahaki. Tēnā anō tātou.

Nau mai, haere atu aku mihi tūārangi ki a koutou ngā tini waka nō ngā pito o te motu, nō ngā hau e whā, e rere atu ana ngā tai ō mihi ki a koutou katoa. Kei aku whakatamarahi ki te rangi, kei aku whakateitei ki ngā whenua, kei aku manu honenga, aku manu puipuiaki kua tau mai rā $i$ te tīkokekoketanga. Kua pakaru mai i te pūrangiaho, i te pūrangitū kua tau, kua tau, kua tau mai rā. Nā koutou ngā riporipo o ngā wai o Te Kōraha i tīhaehae, ka nanao atu ki ngā taura aroha o te teo, o te pou e takoto ana i tōna waka. 
Ka tahuri, ka kite atu i a koutou ngā pū kōrero nō Tauranga moana, Ngāti Pūkenga, Ngāti Ranginui, Ngāi te Rangi. E ai ki te kōrero, ko Tauranga ki tua moana, ko Waikato ki tua whenua, ko Ngāti Mahuta ki te tai hauāuru. Tēnā koutou, tēnā koutou, nau mai.

Whakawhiti atu rā ki a koutou nō te Urewera, anā, i puta mai ai i ngā whāruarua o Ruātoki, ngā uri whakaheke o Te Maunga rāua ko Hinepūkohurangi, te maunga tapu o Maunga Pōhatu, tū mai rā. Kia kōrerohia e au te rua kōiwi, ko Te Waihoanga, e takoto ana te tūpuna rangatira nei a Tūhoe ki Waiharakeke, ka riro i a mātou te tūpuna nei, kia manaakihia e mātou. Tēnā koutou, tēnā koutou, piki mai.

Ki ngā uri whakaheke nō Te Rarawa, nō Ngāti Kahu, te toki o te hoe, te kaha o te au, te kaitiaki, te kaimanaaki, te kaiurungi o te waka o Ngātokimatawhaorua, e te rangatira, Heke-nukumai-ngā-iwi, koutou rā, tēnā koutou, tēnā koutou, kake mai.

Kia pere kau noa taku titiro ki a koutou ngā pūru o te rīki, kua kākāhuria te pae e te kākāriki, tēnā koutou. Ki a koutou, ngā hoa tata, ngā hoa tākaro rīki nō te Karapu Rīki o Taniwharau me ngētehi nō te Tìma Rīki Māori, tēnā koutou, tēnā koutou, hara mai.

Ka whakawhiti atu hoki ki Te Moana-nui-ā-Kiwa, ki a koutou nō ngā moutere o Tonga, o Whìtī, tae atu rā ki ngā matamata huanga nō ngā moutere o Hawai'i, tēnā koutou, tēnā koutou, whakatau mai.

E ngā roopu hoe waka, arā, ngā kaihoe waka ama o te motu, ngā momo tāngata nō ngā whare wānanga, nō ngā wānanga, nō ngā kuratini, nō ngā kura, nō ngā hau e whā, kāore i ārikarika nei ngā mihi manahau, ngā mihi tūranga hākoa ki a koutou katoa, tēnā koutou, tēnā koutou, rarau mai.

$\bar{A}$, kāti ake i konei ngā kōrero whakamihi, ko te taukaea tēnā kei te renarena tonu, mā koutou kē ngā kupu waiwaiā e 
whakatakoto ki ngā rekereke o te koroua nei, heoi anō tāku he wāwāhi i ngā kōrero.

E rau rangatira mā, tēnā koutou, tēnā koutou, huri noa, tēnā koutou katoa. 